\title{
mejoras en el canal de Panamá
}

CHARLES MCG. BRANDL, ingeniero

\section{simopsis}

$533 \cdot 19$

El canal a través del ismo de Panamá es una obra de ingeniería civil que, junto con el de Suez, empiezan ya a clasificarse como obras maestras que señalan el primer jalón del desarrollo de la ingeniería moderna.

otras obras, también importantes, no conservan, sin embargo, la misma reputación que aquéllas, por no seguir un paralelismo con las exigencias en cada época.

El canal de Panamá, con sus exclusas, y angosto paso llamado de la Culebra, ha tenido que afrontar un tráfico creciente de navios que siguen la evolución del tiempo. Para conseguir este paralelismo, tanto el material de explotación como la organización, dragados y mejoras, han tenido que conocer una sucesión ininterrumpida de modificaciones que han permitido conservar, por lo
menos nominalmente, el derecho de paso en especie.

Actualmente, se está ensanchando el canal en algunos trozos para permitir el cruce fácil de barcos de gran tonelaje.

Las obras se están ejecutando a un ritmo acelerado y, con objeto de ganar tiempo, se han repartido en distintas contratas que trabajan simultáneamente.

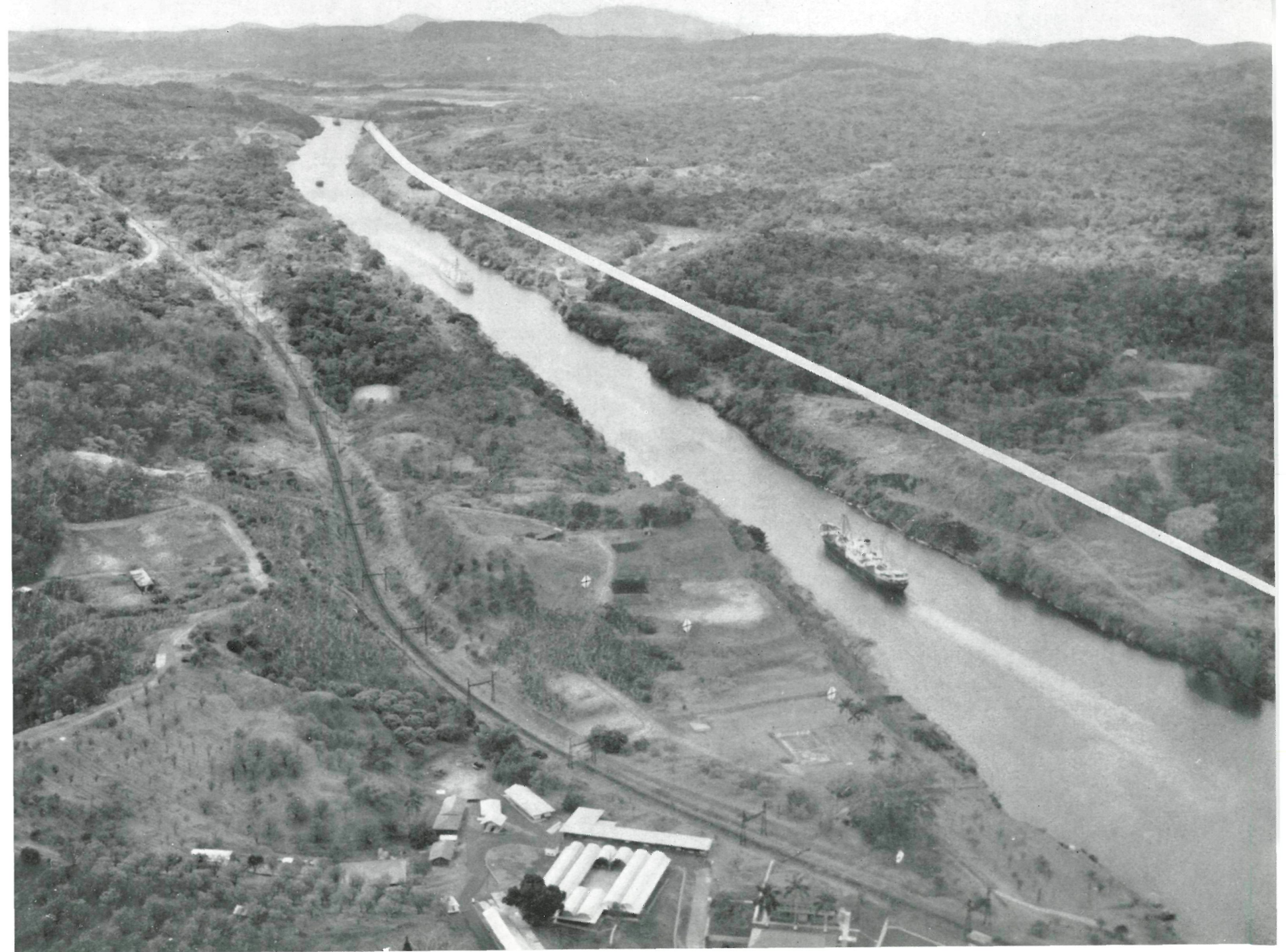



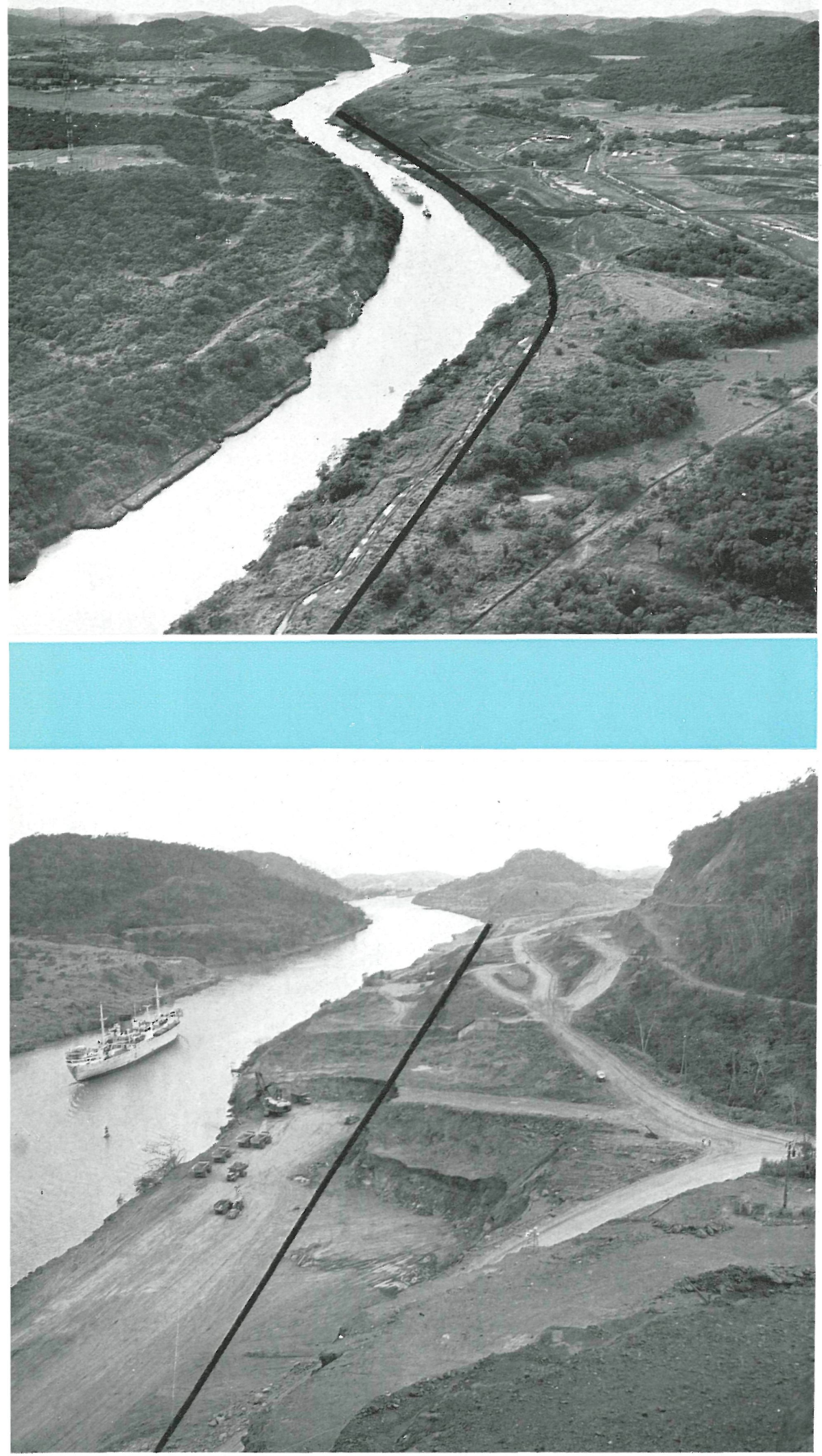

Aspecto de un doble frente escalonado de arranque.

Readaptación de un codo.

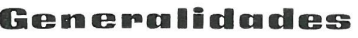

El canal de Panamá se abrió al paso marítimo, entre el Pacífico y el Golfo de Méjico, el 15 de agosto de 1914. Desde el día de su entrada en servicio hasta la actualidad, el canal ha permitido el paso ininterrumpido de barcos de distintas nacionalidades. Este paso ha reducido, en beneficio del mundo entero, el precio de ciertos productos transportados a través del canal, por lo menos, si no en tiempo, en la parte que corresponde al transporte.

Tanto el proyecto como la construcción y conservación del canal han sido objeto de mayor atención. Esto se ha traducido, prácticamente, en los servicios que hasta la actualidad ha venido prestando el canal, labor que hemos de ver reflejada en el crédito de la ingeniería de los EE. UU. y el benévolo interés que esta nación ha prestado al mundo.

Esta acción desinteresada se pone de relieve al tener en cuenta que los derechos de paso iniciales no han sufrido revaloración alguna, a pesar de los cambios constantes de los precios gecambios constantes de los precios ge-
nerales y niveles ascendentes de vida que el mundo viene experimentando.

La consecución de todo esto ha sido posible por las mejoras constantes introducidas en los métodos de conservación y organización general de la explotación del canal.

\section{Ev cerm an}

Resulta notable al considerar el paralelismo que han seguido las mejoras introducidas en los servicios y en el propio canal, del estado de cosas y navegación en el momento de la entrada en servicio de la obra y las actuales condiciones, entre las que resaltan las nuevas embarcaciones y maquinaria auxiliar de dragado, explotación y conservación.

El número, tamaño y velocidad de las embarcaciones han experimentado un cambio sustancialmente notable.

Aunque en términos generales muchas de estas variantes se dejaban prever anticipadamente, esta previsión ha permitido una suave y continua adappermín de los servicios del canal a las circunstancias del momento y aún a previsiones para el futuro. 
Frente de arran. que de una mar-

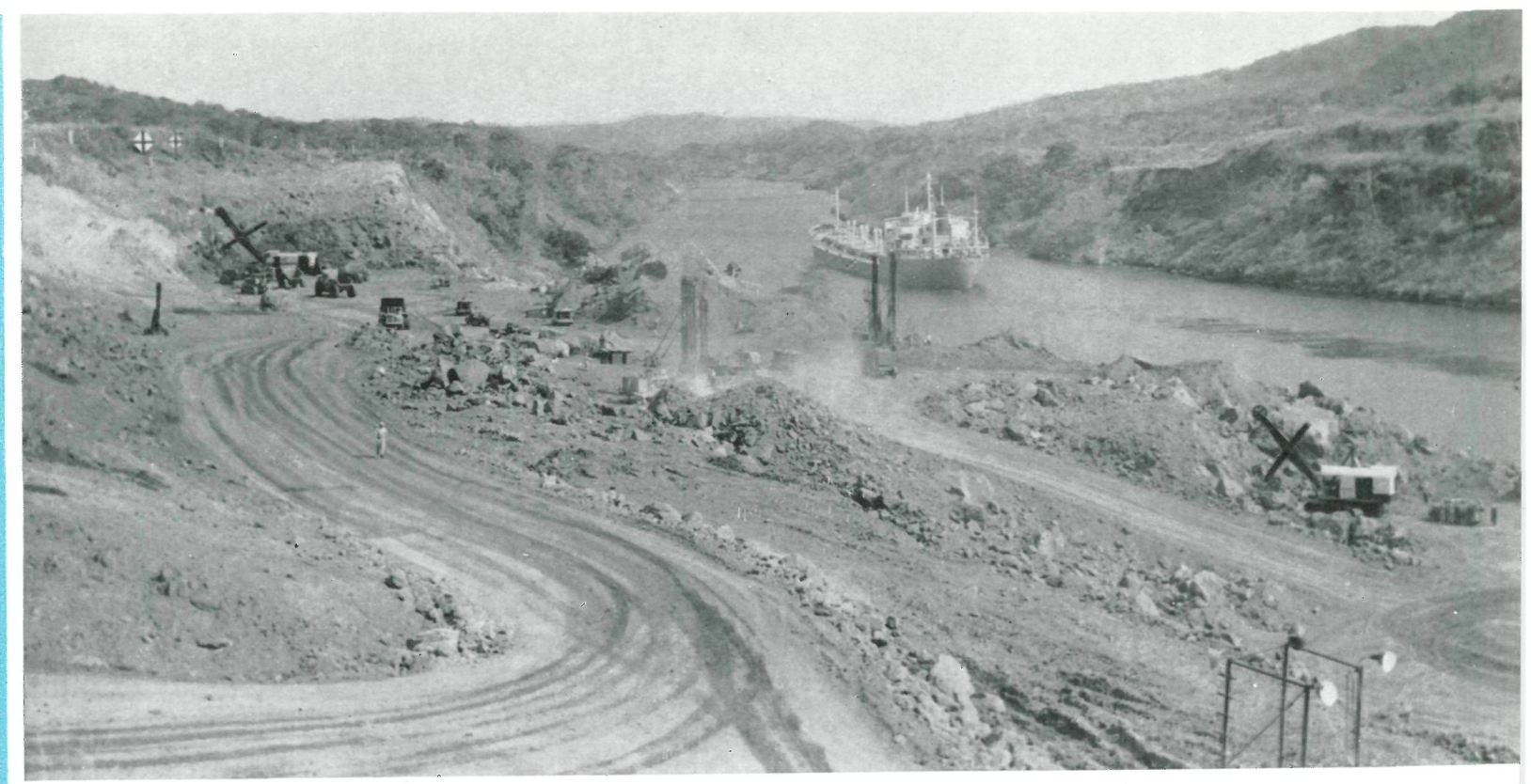

Perforadoras para
el arranque por el arranque
explosivos.

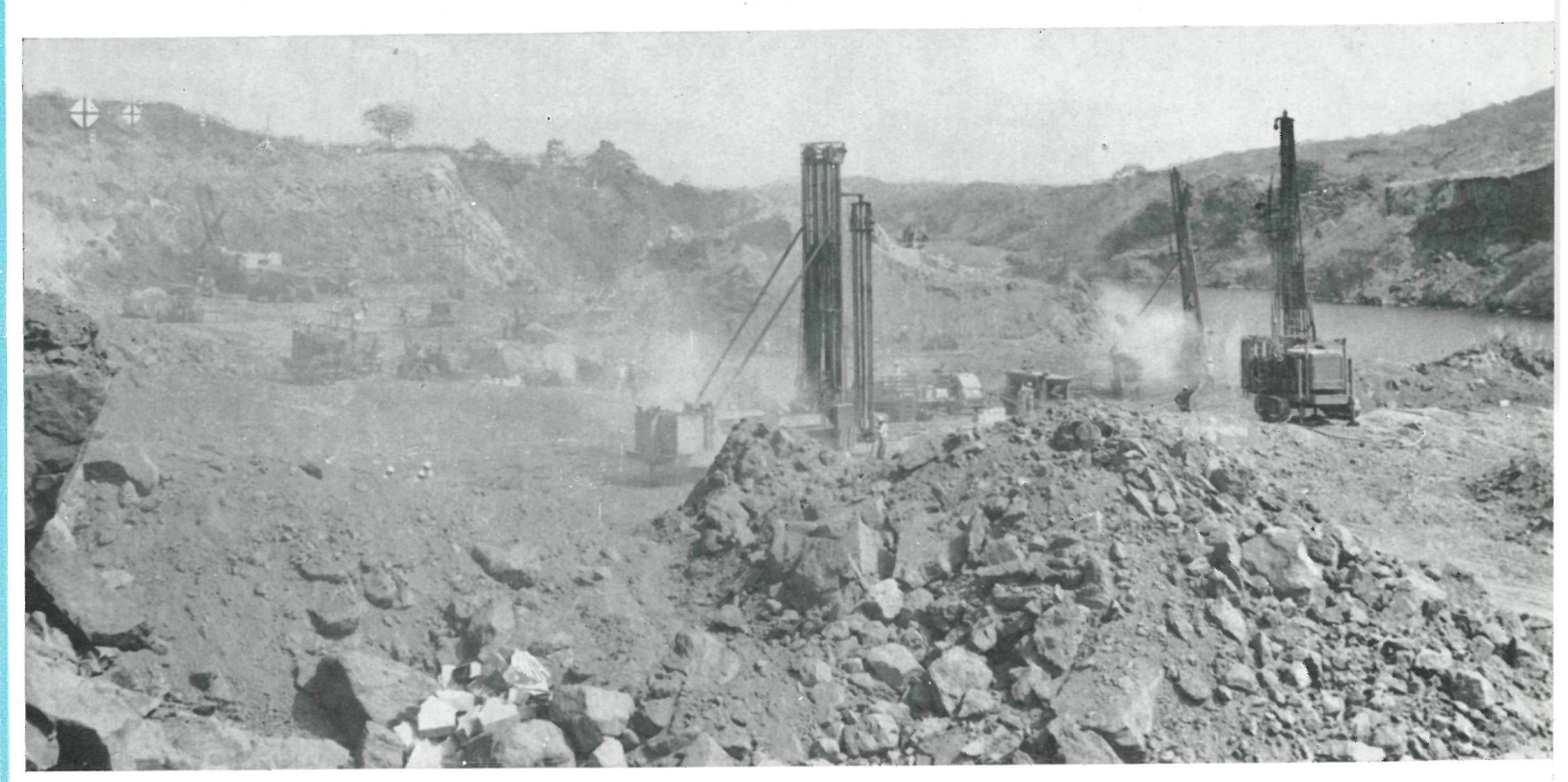

Palas pesadas en

un frente d

arranque.

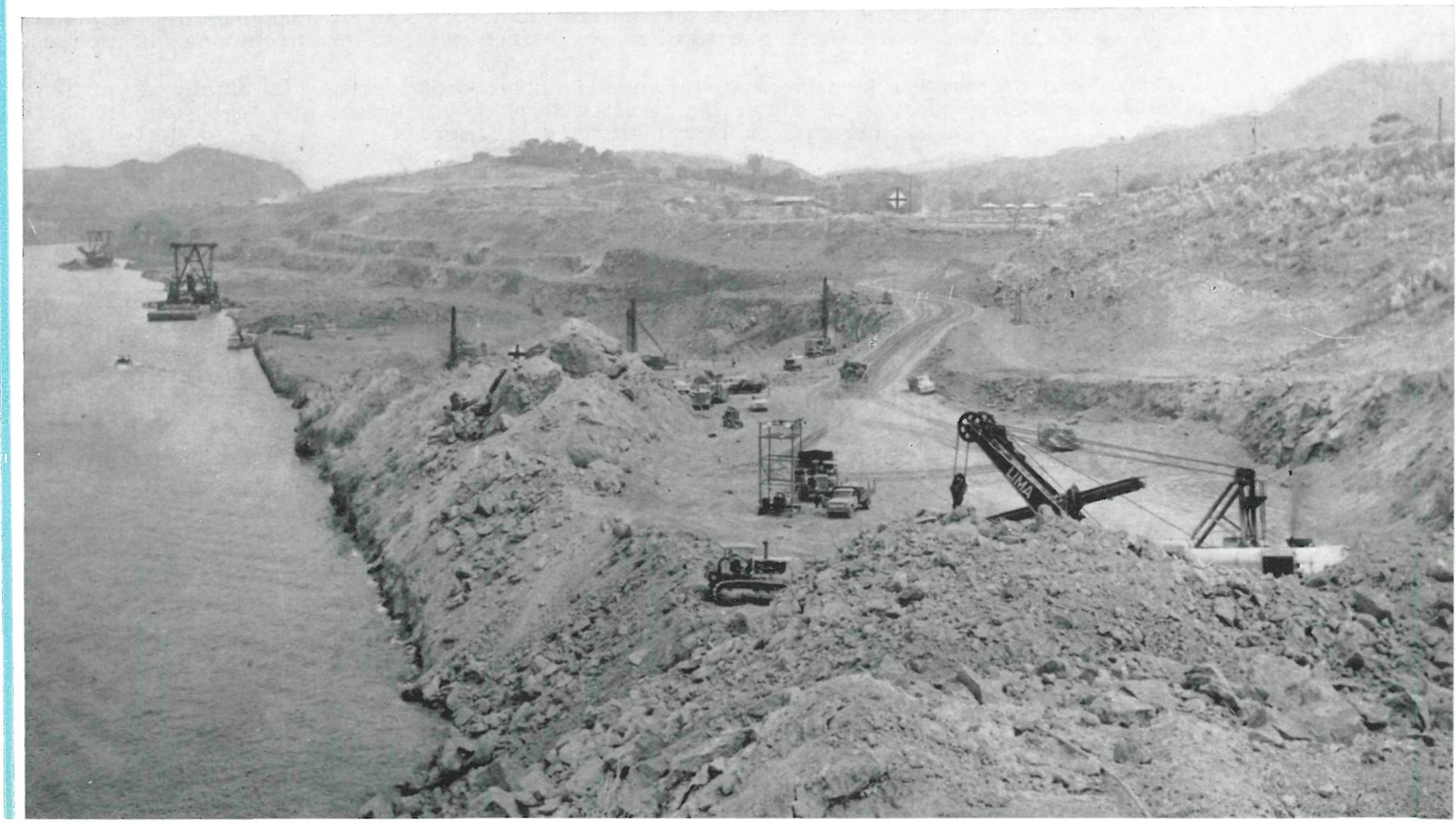




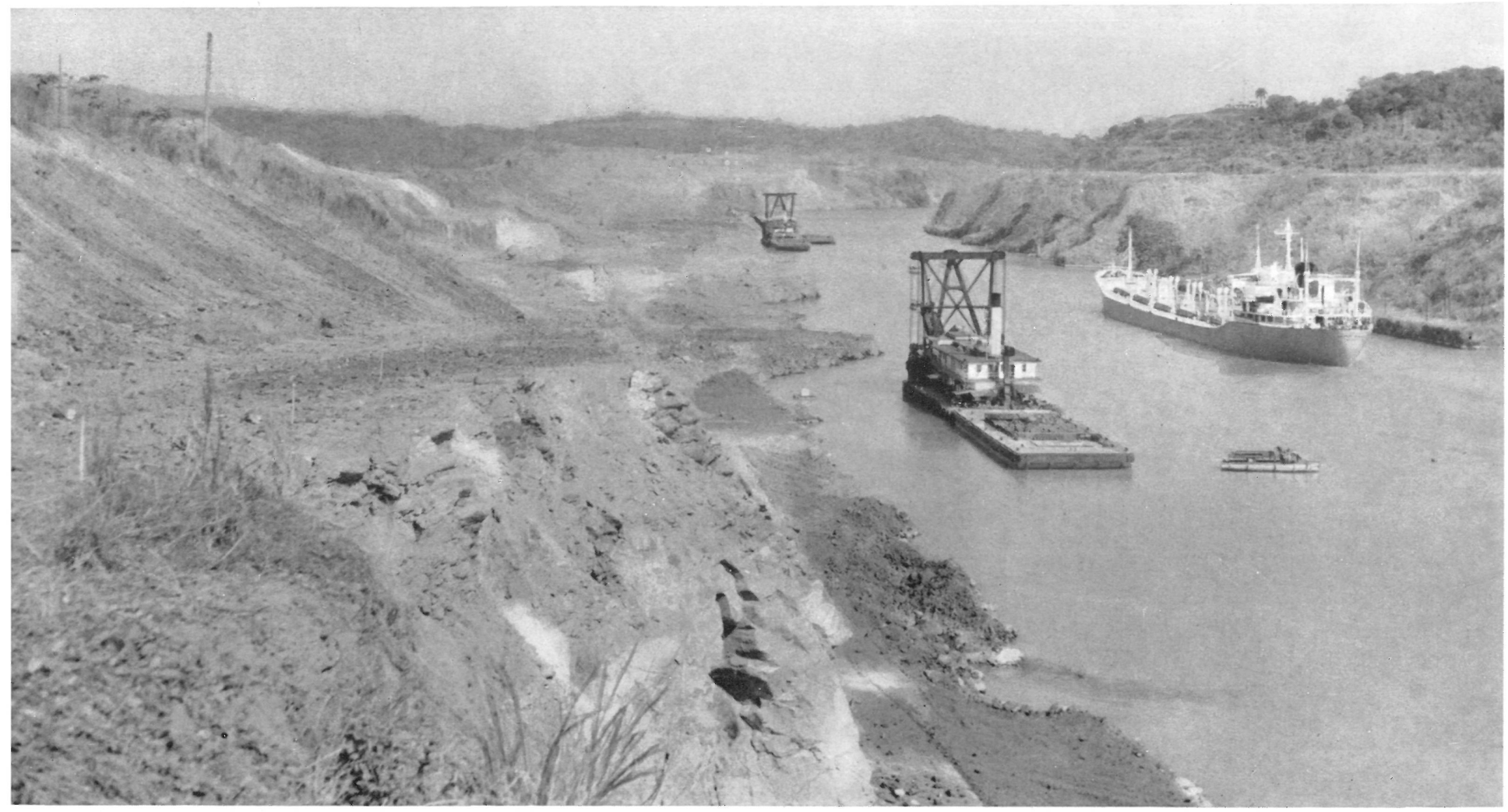

Bareo pasando por la proximidad de una draga.

\section{Mejoras}

El mayor problema que afecta la explotación del canal se centra en el número de barcos que pueden pasar por día. El problema del número tiene su solución en la movilidad y rápida maniobra de las embarcaciones que navegan por el canal, es decir, lograr un paso más rápido en las esclusas, meandros del canal y coordinación de fechas en la navegación de altura para evitar las colas o esperas en las bahías de las dos entradas del canal.

Para dar mayor capacidad al canal se han iniciado una serie de ensanchamientos, así como también la sustitución de las actuales locomotoras que tiran de los barcos para su movimiento en las esclusas.

Con objeto de permitir la navegación durante la noche se ha instalado un alumbrado artificial. Otra instalación notable, electrónica, consiste en captar las características de los barcos que se aproximan al canal con objeto de ofrecerles el momento óptimo para su paso a través del mismo.

De todas estas mejoras la más costosa, sin duda alguna, es la del ensanchamiento de los $12 \mathrm{~km}$ que constituyen el paso del canal a través de la divisoria, tramo que se conoce con el nombre "Gaillard Cut» o paso de la Culebra. Este paso, de sólo $92 \mathrm{~m}$ de anchura, ha presentado grandes dificultades a los barcos muy cargados, de gran calado, ya que este tipo de embarcaciones navegaba en este trozo a una velocidad muy lenta.

Estos barcos, cuyo número iba aumentando cada día, suponían un grave embotellamiento en estas zonas, porque no dejaban pasar a otras embarcaciones más ligeras que, de esta forma, se veían obligadas a esperar.

\section{Ejecución de mejomis}

Las obras de ensanchamiento del paso de la Culebra se iniciaron hace ya muchos años y a un ritmo cuya terminación se preveía para algunos años más.

El aumento continuo de la navegación a través del canal no podía esperar la terminación de las mejoras emprendidas a un largo plazo. Esto obligó, en 1954, a conceder una primera contrata de obras, y, a partir de esta fecha hasta la actualidad, el número de contratas ha ido aumentando hasta llegar a 12.

La anchura inicial de $92 \mathrm{~m}$ en el paso de la Culebra se ha llevado a $140 \mathrm{~m}$, y se espera ver terminada la mejora en 1966. Para tener una idea del volumen de obra que se ha de realizar, puede adelantarse que el movimiento de tierras y roca en superficie será del orden de $20.000 .000 \mathrm{~m}^{3}$ y unos $11.000 .000 \mathrm{~m}^{3}$ en dragados de profundización. 
Pega de arranque en un frente. Explosión de la pega de la figura anterior.

Nótese el magnífico troceo después de la pega.

Para conseguir este movimiento de tierras habrán de perforarse, aproximadamente, $1.850 .000 \mathrm{~m}$ de barrenos que consumirán unas 8.400 toneladas de dinamita para su carga y explosión. Los explosivos de superficie tienen un 40 por 100 de dinamita y los 40 por 100 de dinamita y los
subacuáticos un 60 por 100 , subacuáticos un 60 por 100 , pero envueltss con un material especial impermeable, Las pegas se har realizado con redetonadores conectados, por medio de lír. ss, a explosores eléctricos.

A pesar de estos importantes trabajos en curso, la navegación no ha sido interrum. pida, para lo cual na sido ne cesario acoplarse ceñidamente a un plan general riguroso de coordinación entre los servicios de navegación y los de obras.

Para el movimiento y arranque de tierras en la superficie se ha venido empleando un equipo moderno de maqui naria auxiliar pesada. En los trabajos de profundización y dragado, la maquinaria auxiliar va montada sobre pontones, y el escombro, cargado sobre barcazas de fondo basculante, es transportado a fondos profundos previamente elegidos.

En esta zona tropical el pe. ríodo de lluvias es de unos ochn meses del año; pues bien, a pesar de esto, los trabajos han venido realizándo. se por las distintas contratas de acuerdo con los planes de acuerdados.

Los trabajos de mejoras emprendidos se van desarrollando con el ritmo y regula. ridad previstos. Por tanto, el ridad previstos. Por tanto, el canal seguirá prestando sus lerada que seguirá de cerca y paralelamente las exigen cias, cada día crecientes, de la navegación a través de es. te canal de tanta trascendenmundo y gran sig. grandes y ambiciosas obras de ingeniería civil.
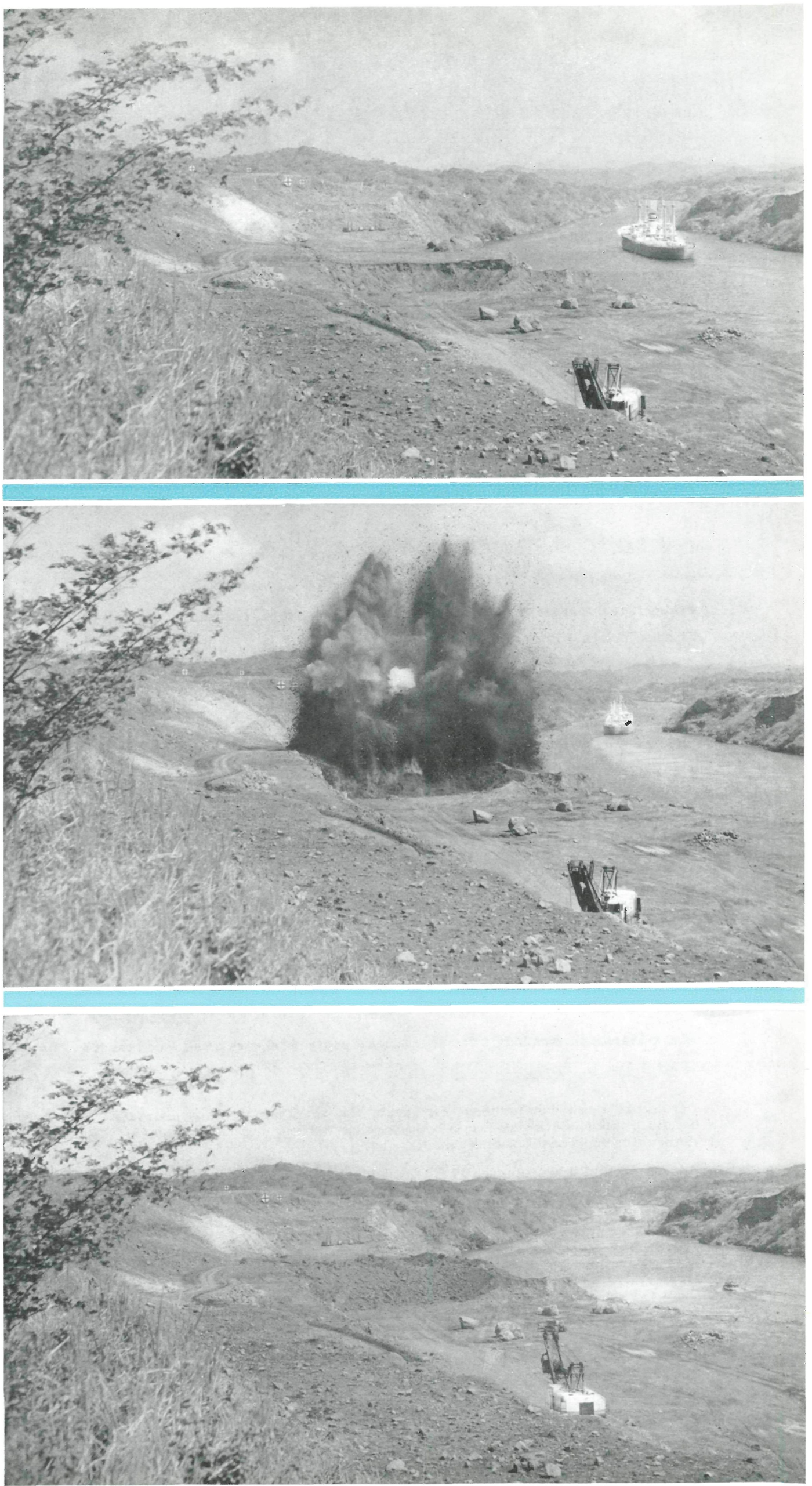


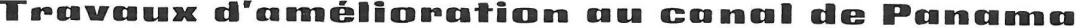

Charles Mc. G. Brandl, Prof. Ing.

Le canal de Panama, comme celui de Suez, est un ouvrage de génie civil qui, déjà, se classe parmi les grands chefs-d'oeuvre qui marquent les premiers jalons du développement du génie moderne.

D'autre ouvrages, d'égale importance, ne gardent pourtant pas cette même réputation, parce qu'ils ne suivent pas le même parallélisme avec les exigences de chaque époque.

Le canal de Panama, avec ses écluses et son étroit passage appelé «la Couleuvre», a dû faire face à un trafic croissant de navires dont les proportions suivent l'évolution du temps. Pour arriver à ce parallélisme, le matériel d'exploitation aussi bien que l'organisation, drainage et améliorations ont dû subir une série inin. terrompue de modifications qui ont permis de conserver, au moins nominalement, le droit de passage.

A l'heure actuelle on procède à l'élargissement de quelques sections du canal afin de faciliter le croisement des navires de grand tonnage.

Les travaux se réalisent à un rythme accéléré et, afin de gagner du temps, ont été répartis entre plusieurs entreprises qui travaillent simultanément.

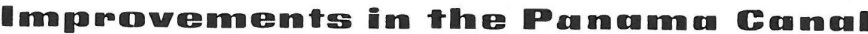

Charles Mc. G. Brandl, professor of engineering.

The Panama Canal, together with the Suez Canal, is an engineering accomplishment which is now regarded as a master work marking the initiation of modern civil engineering.

Other projects, also of great magnitude, do not seem to maintain the reputation of the above two, because they cannot continue to serve their function with sustained usefulness in the new circumstances of today.

The Panama Canal, with its limited dock capacity, and the narrow, so called Serpent, canal, has had to cope with a continuously increasing volume of shipping traffic; a natural consequence of commercial evolution. In order to keep up with these increasing demands, it has been necessary to carry out an almost uninterrupted series of modifications and improvements, affecting both the method of operation and organisation, and the actual nature of the canal. Thanks to these alterations the Panama Canal has continued to maintain, at least nominally, an adequate standard of functional efficiency.

At present the canal is being widened over certain sections to enable the easy passage of ships of great displacement. The work is being done very rapidly, and in order to gain time, it has been distributed to various contractors simultaneously.

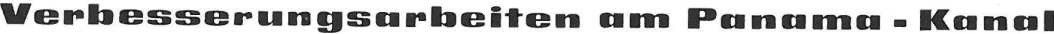

Charles Mc. G. Brandl, Prof.-Ing.

Der Kanal durch den Isthmus von Panama ist ein Werk des Ingenieurbaues, der, zusammen mit dem Suez, bereits beginnt, als Meisterwerk betrachtet zu werden, der den ersten Markstein der Entwicklung des modernen Ingenieurbaues kennzeichnet.

Andere ebenfalls bedeutende Werke jedoch erhalten nicht den gleichen Ruf wie jene, da sie keinem Gleichlauf mit den Forderungen in jeder Zeit folgen.

Der Panama-Kanal mit seinen Besonderheiten und dem engen, so genannten Schlangen-Durchgang hat sich einem steigenden Schiffsverkehr, welcher der Entwicklung der Zeit folgt, gegenübergesehen. Um diesen Gleichlauf zu erreichen, mussten sowohl das Betriebsmaterial als auch die Organisation, die Baggerung und Verbesserung, eine ununterbrochene Aufeinanderfolge von Aenderungen anerkennen, die es erlaubt haben, das Durchfahrtsrecht wenigstens nominell aufrechtzuerhalten.

Gegenwärtig wird der Kanal an einigen Stellen verbreitert, um das leichte Kreuzen von Schiffen mit grossem Tonnengehalt zu erlauben.

Die Arbeiten werden in einem beschleunigten Rhythmus durchgeführt, und damit Zeit gewonnen werde, wur. den sie auf verschiedene Verträge, die gleichzeitig arbeiten, aufgeteilt. 